\title{
Impact of praziquantel mass drug administration campaign on prevalence and intensity of Schistosoma haemamtobium among schoolchildren in Bahi district, Tanzania
}

\author{
SIMON A. CHAULA ${ }^{1}$ and DONATH S. TARIMO ${ }^{2 *}$ \\ 'Department of Biomedical Sciences, School of Medicine, University of Dodoma, Tanzania \\ ${ }^{2}$ School of Public Health and Social Sciences, Muhimbili University of Health \& Allied Sciences, Dar es Salaam, \\ Tanzania
}

\begin{abstract}
As part of the Tanzania National Schistosomiasis Control Programme, Bahi district in central Tanzania, received two annual rounds of praziquantel mass drug administration (MDA) to control urinary schistosomiasis in schoolchildren. The objectives of this study were to assess the impact of the two rounds of MDA on prevalence and intensity of Schistosoma haemamtobium and the impact of MDA campaigns on knowledge of urinary schistosomiasis, safe water use and contact with potentially unsafe water bodies. A quantitative crosssectional study was carried out among schoolchildren in March and April, 2013. A structured questionnaire was used to collect information on MDA uptake, knowledge of schistosomiasis, sources of water for domestic and other uses. Urine samples were collected from each pupil to examine prevalence and intensity of $S$. haematobium. Transmission of schistosomiasis was assessed by sampling Bulinus spp snails for cercarial shedding. Uptake of MDA was $39.5 \%$ in 2011 and $43.6 \%$ in 2012. Prevalence of S. haematobium significantly dropped by $50.0 \%$ from $26 \%$ in 2011 to $15 \%$ in $2012(p=0.000)$. Prevalence of $S$. haematobium was significantly low in MDA participating (3.1\%) than non-participating $(28.5 \%)$ schoolchildren ( $p=0.000)$. MDA campaigns had significant impact on knowledge of the disease $(p=0.02)$ and borderline impact on safe water use $(p=0.04)$ but had no impact on avoidance of contact with unsafe water bodies $(p=0.06)$. Bulinus spp. snails were found shedding schistosome cercariae indicating environmental contamination with viable $S$. haematobium eggs. In conclusion, though MDA significantly reduced prevalence of S. haematobium, uptake was below $50.0 \%$ and below the World Health Assembly resolution 54.19 target of $75.0 \%$ for 2010. Non-participation in MDA was the likely source of $S$. haematobium eggs in the environment hence the observed $15.0 \%$ prevalence of $S$. haematobium infection; and cercarial shedding Bulinus spp. snails indicating continuity of transmission hence the need for further health promotion campaigns.
\end{abstract}

Keywords: urinary schistosomiasis, schoolchildren, mass drug administration, praziquantel, Tanzania

\section{Introduction}

Urinary schistosomiasis is a chronic disease caused by infection with the parasitic trematode known as Schistosoma haematobium. Common early signs of $\mathrm{S}$. haematobium infection include microscopic and visible haematuria that precede the development of urological abnormalities such as urinary bladder calcification, deformed ureters and hydroneprosis; significantly more common in schoolchildren with higher infection intensities (WHO, 2002). It is estimated that 70 million individuals out of 682 million experience haematuria and 32 million people dysuria associated with $S$. haematobium infection in Sub-Saharan Africa; 18 million suffer bladder wall pathology and 10 million hydronephrosis (WHO, 2012). On a nationwide scale, available data show that all administrative regions of Tanzania mainland have some level of schistosomiasis infection, ranging from $12.7 \%$ to 87.6\%; however, it is most prevalent along the coast of the Indian Ocean and shores of Lake Victoria (MoHSW, 2012). The levels of infection are known to be highest in children of school age (WHO 2002), thus, the national goal of urinary schistosomiasis control has been to reduce prevalence and intensity of infection among schoolchildren by Praziquantel MDA. In so doing assuming parasitological cure, the children will stop shedding S. haematobium eggs in the environment

\footnotetext{
*Correspondence: Donath S. Tarimo; E-mail: dontarimo@gmail.com
} 
(Stoddard et al., 2013). This will lead to a reduction in S. haematobium eggs contamination of fresh water bodies with Bulinus spp. snails' intermediate hosts (SCI/MoHSW, 2010). As treatment does not prevent against subsequent infection, if water contact is continued, re-infection can take place relatively quickly. However, the pathology that the eggs induce is somewhat diminished by a reduction in their production such that future morbidity is averted (Stothard et al., 2013). Thus Praziquantel MDA to children of school age must be accompanied with the provision of Information Education Communication (IEC) and Behaviour Change Communication (BCC) towards inducing community participation in the programme, personal hygiene and sanitation, and the reduction of contact with fresh water bodies with cercarial shedding Bulinus spp. snails by the provision of safe water supplies.

In Tanzania, school-based Praziquantel MDA campaign was adopted by the MoHSW and launched in July 2005. Phase I MDA implementation was conducted in 2005 (November and December) and 2007 in 11 regions, while Phase II was conducted in 2008 in 6 regions highly endemic for S. haematobium (MoHSW, 2012). Communities were selected for MDA on the basis of prevalence of schistosomiasis: in schools with prevalence $<10 \%$, PZQ is made available in local health facilities; prevalence $10-50 \%$ annual Praziquantel MDA is given to all school age-children and prevalence $>50 \%$ annual Praziquantel MDA given to all school age children plus other high-risk groups in the community (WHO, 2002).

The Dodoma region in central Tanzania was involved in the Praziquantel MDA campaigns during Phase II implementation covering six districts, including Bahi district. In the Bahi district, before the MDA campaigns the prevalence of visible blood in urine (indicative of $\mathrm{S}$. haematobium infection) among children of school-age was $26.0 \%$ equivalent to an average of $30.0 \%$ microscopic prevalence of $S$. haematobium infection representing a community category for annual Praziquantel MDA to all school age-children (WHO, 2002; SCI/MoHSW, 2010). Monitoring and evaluation of the performance of MDA campaigns such as coverage and therapeutic impact are important component of the intervention (MoHSW, 2012). Therefore there was a need to assess the proportion of schoolage children who participated in the MDA campaigns and impact of the two rounds of annual Praziquantel MDA on the prevalence and intensity of S. haematobium infection.

Coverage is an important component of monitoring and evaluation as children who did not participate in MDA campaigns are likely to be the source of shedding $S$. haematobium eggs in the environment and contamination of fresh water bodies with Bulinus spp. snails' intermediate hosts (SCI/MoHSW, 2010; Stoddard et al., 2013). If water contact is continued with these water bodies potentially infested with cercariae shedding Bulinus spp. snails, re-infection can take place relatively quickly (Stoddard et al., 2013). Thus there was need to assess the extent to which the MDA advocacy campaigns changed community knowledge on urinary schistosomiasis in terms of their roles in transmission, contact with potentially unsafe water sources and therefore re-infection, recognition of morbidity (blood in urine) and their participation in MDA campaigns.

This study assessed coverage and the therapeutic impact of two rounds of Praziquantel MDA on the prevalence and intensity of S. haematobium among primary schoolchildren and the impact of MDA advocacy campaigns on knowledge and practices related to S. haematobium transmission in Bahi district in central Tanzania.

\section{Materials and Methods}

\section{Study area}

A quantitative cross-sectional survey of school children was conducted in Bahi district in central Tanzania from March to April 2013. Bahi is one of the six districts in Dodoma region in which Praziquantel MDA campaigns had been carried out. Bahi district is at latitude: $5^{\circ} 55^{\prime} 14^{\prime \prime} \mathrm{S}$ longitude: 
$35^{\circ} 17^{\prime} 22^{\prime \prime} E$ with a population of 221,645 people and total area of $5,498 \mathrm{~km}^{2}$ with several water bodies such as rivers, ponds, marshy swamps and irrigated rice fields. People are at risk of infection with $S$. haematobium due to agricultural, domestic and recreational activities which expose them to water bodies potentially infested with cercariae shedding Bulinus spp. snails. Lack of hygiene in urine disposal and play habits make schoolchildren especially at risk to infection. The prevalence of visible blood in urine (indicative of S. haematobium infection) among children of school-age was $26.0 \%$ before MDA campaigns (SCI/MoHSW, 2010) representing a community category for annual Praziquantel MDA to all school age-children (WHO, 2002)

\section{Sample size and sampling procedure}

Primary schoolchildren attending classes during the time of study constituted the study population.

The study sample size was derived using the formulae: $N=\left(\frac{z}{d}\right)^{2} p(1-p) f$

Where, $\mathrm{N}=\quad$ the desired sample size

$z=\quad$ standard deviation (1.96) which corresponds to $95 \%$ confidence interval.

$p=$ baseline prevalence of schistosomiasis in the study area $(26 \%)=0.26$

$\mathrm{f}=\quad$ design effect factor (1.5)

$\mathrm{d}=$ marginal error $(5 \%)=0.05$

Thus, $\mathrm{N}=\left(\frac{1.96}{0.05}\right)^{2} \times 0.26(1-0.26) \times 1.5=444 ;$ adding $10 \%$ for non-responses, drop outs and missing data, the sample size became; $(0.1 \times 444)+444=488$ schoolchildren.

Bahi district was purposively chosen as one among districts with moderate to high transmission and in which Praziquantel mass drug administration has been conducted (MoHSW, 2012). Multistage random sampling technique was used. At first stage wards were randomly selected, at second stage 5 primary schools (Bahi Misheni, Bahi Sokoni, Bahi Makulu, Nagulo Bahi and Uhelela) were randomly selected and at third stage schoolchildren were randomly selected from class III to VII to get the required sample size. The ages of the children were obtained from the registry at the first year of enrolment; and extrapolated to the year at the current class.

\section{Data collection}

A questionnaire interview was carried out with school children to assess participation in MDA advocacy campaigns and uptake of Praziquantel MDA in 2011 and 2012 rounds. Knowledge on the disease was assessed by interviewing the children on the modes of transmission (contact with unsafe water sources potentially infested with cercariae), symptoms of the disease (blood in urine, painful urination and suprapubic pain), availability of treatment (can be treated by modern medicine) and control measures (avoid urination in water sources, avoid contact with unsafe water sources, use of safe source of water for domestic use from taps and wells, participation in MDA advocacy campaigns). Water contacts were assessed by interviewing the children on the reasons for coming into contact with a water source and the sources of water for domestic use and other activities.

Urine samples were collected from each pupil to determine the prevalence and intensity of $S$. haematobium infection. The children were asked to submit terminal stream urine in $50 \mathrm{ml}$ screw top clean dry plastic containers. The specimens were transferred to University of Dodoma, Parasitology laboratory under formalin fixation as preservative for microscopic examination of $\mathrm{S}$. haematobium eggs. The presence of $S$. haematobium eggs was determined by urine centrifugation method. Urine deposits were placed on clean labelled glass slides, stained with Lugol's iodine and microscopically examined under $\mathrm{X}_{10}$ and $\mathrm{X}_{40}$ magnifications. Intensity of infection for each positive case was established using urine filtration method, by straining $10 \mathrm{mls}$ of positive urine samples on nucleopore filters, stained with Lugol's iodine, covered by cover glass and placed on the microscope for examination under $\mathrm{X} 40$ magnification. Egg counting was recorded as number of eggs per 10mls of 
urine (WHO, 1991).

To establish continued transmission, fresh water snails from water bodies within the study area were sampled and examined for cercarial shedding. Snails of the Bulinus spp. were sampled from water bodies around the studied schools. These were collected in plastic bowls and lined by grass cover from the same water body and transported to the Parasitology laboratory at the University of Dodoma. The snails were morphologically identified as Bulinus spp using a field guide described by Kristensen (1987). The cercarial shedding experiment was carried out by placing the snails in small glass containers with $20 \mathrm{mls}$ of warm water and were incubated on indirect sunlight at different time intervals and observed for cercarial shedding. From Bahi Centre pond we collected 14 snails, from Nagulo Bahi 16 snails and from Uhelela pond 16 snails. Then 2 snails from each pond were incubated under indirect sun light for cercarial shedding. Those from Bahi Centre pond were incubated from 10:00 AM to 12:00 (2 hours); those from Nagulo Bahi pond were incubated from 10:00 to 2:00 (4 hours) while those from Uhelela pond were incubated from 10:00 AM to 1:00 (3 hours). Water from glass containers was centrifuged, and deposits examined by light microscope under $\mathrm{X}_{10}$ and $\mathrm{X}_{40}$ magnification, for the presence of schistosome cercariae. All brevifurcate apharyngeate distome cercariae were identified as those of S. haematobium by morphology as previously described by Fransden \& Christensen (1984).

\section{Data analysis}

During and after collection, data were coded, classified and edited, for adjustment of any missing information and correction of outliers. Data were cleaned, entered in the computer and analysed by SPSS version 16.0 software. Descriptive analysis was done to calculate coverage of Praziquantel MDA uptake, microscopic prevalence and intensity of S. haematobium infection. The baseline blood in urine prevalence of $26.0 \%$ was extrapolated to an average microscopic prevalence of $30.0 \%$ (WHO, 2002). Prevalence and intensity of $S$. haematobium infection before and after two rounds of MDA was compared. Knowledge was assessed on a scale of 9 points score with categories: 1-3 considered as low, 4-6 medium and 7-9 as high knowledge. The categories followed correct mentioning of the modes of transmission (contact with cercariae infested waters), symptoms of the disease (blood in urine, painful urination and suprapubic pain), availability of treatment (can be treated by modern medicine) and control measures (avoid urination in water sources, avoid contact with unsafe water sources, use of safe source of water such as taps and wells for domestic use, participation in MDA advocacy campaigns) to constitute 9 points score. The chi-square test was used to compare proportions between groups and to examine associations between variables at a significance level of 0.05 .

\section{Ethical considerations}

The study was ethically cleared by the Muhimbili University of Health \& Allied Sciences Institutional Review Board. Permission to carry out the study was obtained from the District authorities and the head teachers of the schools. Written informed consent was obtained from parents / guardians through a letter from the class teacher sent to them by each child. Results of urine microscopy were communicated to all children by their class teacher and those found infected were referred to the nearest health facility for treatment.

\section{Results}

\section{Proportion of school children who received Praziquantel}

A total of 488 schoolchildren (mean age $=13 \pm 2.69$ years) were enrolled in the study (Table 1 ). Of these, 244 (50.0\%) were males. 
Table 1: Characteristics of the study sample $(\mathrm{N}=488)$

\begin{tabular}{llll}
\hline Attributes & & Number & Percentage (\%) \\
\hline Sex & Male & 244 & 50.0 \\
Age in (years) & Female & 244 & 50.0 \\
& $8-11$ & 129 & 26.4 \\
School & $12-15$ & 256 & 52.5 \\
& $16-19$ & 103 & 21.1 \\
& Bahi Misheni & 100 & 20.5 \\
& Bahi Sokoni & 100 & 20.5 \\
& Bahi Makulu & 96 & 19.7 \\
& Nagulo Bahi & 96 & 19.7 \\
& Uhelela & 96 & 19.7 \\
\hline
\end{tabular}

All the selected primary schools were involved in MDA campaigns of 2011 and 2012. Out of the 488 enrolled children, 193 (39.5\%) versus 213 (43.6\%) received Praziquantel in 2011 and 2012 respectively (Table 2 ). Less than a third (29.9\%) received Praziquantel in both rounds.

Table 2: Praziquantel administration by School $(\mathrm{N}=488)$

\begin{tabular}{lllll}
\hline School & Number & \multicolumn{2}{l}{ Received praziquantel in MDA } \\
& & $\mathbf{2 0 1 1}$ & $\mathbf{2 0 1 2}$ & $\mathbf{2 0 1 1}$ and 2012 \\
\hline Bahi Misheni & 100 & $15(7.7 \%)$ & $26(12.2 \%)$ & $15(10.3 \%)$ \\
Bahi Sokoni & 100 & $45(23.3 \%)$ & $42(19.7 \%)$ & $23(15.7 \%)$ \\
Bahi Makulu & 96 & $58(30.1 \%)$ & $64(30.0 \%)$ & $53(36.3 \%)$ \\
Nagulo Bahi & 96 & $43(22.3 \%)$ & $44(20.7 \%)$ & $29(19.9 \%)$ \\
Uhelela & 96 & $32(16.6 \%)$ & $37(17.4 \%)$ & $26(17.8 \%)$ \\
Total & 488 & $193(39.5 \%)$ & $213(43.6 \%)$ & $146(29.9 \%)$ \\
\hline
\end{tabular}

\section{Prevalence and intensity of S. haematobium infection}

All of the study participants were screened for S. haematobium infection; $73(15.0 \%)$ had $S$. haematobium infection. About half (46.6\%) of the children found to be infected with S. haematobium were from Nagulo Bahi primary school (Table 3). Of those infected, 57 (78.1\%) had light infection, 9 $(12.3 \%)$ moderate infection and 7 (9.6\%) heavy infection.

Table 3: Prevalence and Intensity of S. haematobium infection by School $(\mathrm{N}=488)$

\begin{tabular}{lllllll}
\hline School & Number & \multicolumn{2}{l}{$\begin{array}{l}\text { S. haematobium eggs present } \\
\text { Yes }\end{array}$} & $\begin{array}{l}\text { Intensity } \\
\text { Light }\end{array}$ & Moderate & Heavy \\
\hline Bahi Misheni & 100 & $8(11.0 \%)$ & $92(22.2 \%)$ & $6(10.5 \%)$ & $0(0.0 \%)$ & $2(28.6 \%)$ \\
Bahi Sokoni & 100 & $7(9.6 \%)$ & $93(22.4 \%)$ & $4(7.0 \%)$ & $3(33.3 \%)$ & $0(0.0 \%)$ \\
Bahi Makulu & 96 & $12(16.4 \%)$ & $84(20.2 \%)$ & $9(15.8 \%)$ & $2(22.2 \%)$ & $1(14.3 \%)$ \\
Nagulo Bahi & 96 & $34(46.6 \%)$ & $62(14.9 \%)$ & $26(45.6 \%)$ & $4(44.4 \%)$ & $4(57.1 \%)$ \\
Uhelela & 96 & $12(16.4 \%)$ & $84(20.2 \%)$ & $12(21.1 \%)$ & $0(0.0 \%)$ & $0(0.0 \%)$ \\
Total & 488 & $73(15.0 \%)$ & $415(85.0 \%)$ & $57(78.1 \%)$ & $9(12.3 \%)$ & $7(9.6 \%)$ \\
\hline
\end{tabular}

Prevalence of S. haematobium infection was significantly low (3.1\%; 8/260) among school-age children involved in MDA advocacy campaigns than among schoolchildren not involved in the MDA advocacy campaign (28.5\%; 65/228 $\left(X^{2}\right.$ test $=61.761, \mathrm{df}=1: p$-value $\left.=0.000\right)$. Schoolchildren not involved in MDA advocacy campaigns were significantly more likely to have heavy $(21.5 \%)$ to moderate $(3.9 \%) \mathrm{S}$. haematobium infection than those involved in MDA advocacy (3.1\% and $0 \%$, respectively) $\left(X^{2}\right.$ test $=$ 
64.234, $\mathrm{df}=3: p=0.000$ ). None of the children involved in MDA advocacy had moderate or heavy infection intensity.

\section{School children and access to safe water supplies}

All of the school children reported to have more than one sources of water for domestic use (Table 4). The most frequently reported source of safe water for domestic use were wells (76.6\%) and taps $(62.3 \%)$.

Table 4: Distribution of study sample by source of water for domestic use $(\mathrm{N}=488)$

\begin{tabular}{lll}
\hline Water source & $\begin{array}{l}\text { Response* } \\
\text { Yes }\end{array}$ & No \\
\hline Tap & $304(62.3 \%)$ & $184(37.7 \%)$ \\
Well & $374(76.6 \%)$ & $114(23.4 \%)$ \\
Pond & $226(46.3 \%)$ & $262(53.7 \%)$ \\
Dam & $119(24.4 \%)$ & $369(75.6 \%)$ \\
River & $284(58.2 \%)$ & $204(41.8 \%)$ \\
Streams & $65(13.3 \%)$ & $423(86.7 \%)$ \\
\hline
\end{tabular}

*= Multiple response analysis

A total of 219 (84.2\%) and 206 (90.4\%) children involved and not involved in MDA campaign, respectively, were using safe water. The involvement in MDA advocacy campaigns was not associated with use of safe sources of water for domestic use $\left(X^{2}\right.$ test $\left.=4.047, d f=1: p=0.054\right)$. Generally all 488 had contact with water bodies and among them, 279 (57.2\%) had contact with rivers, 183 (37.5\%) with ponds, 255 (52.3\%) with dam and 263 (53.9\%) with irrigated rice fields.

\section{Knowledge about urinary schistosomiasis disease}

Knowledge about the disease was categorized into 3 levels on a scale of 9 points score with categories: 1-3 considered as low, 4-6 medium and 7-9 as high knowledge as described in the methodology. The large majority (90.6\%) of the school children had high knowledge about urinary schistosomiasis (Table 5). Involvement in Praziquantel MDA advocacy campaigns was significantly associated with having high level of knowledge about urinary schistosomiasis $(p=0.021)$.

Table 5: Relationship between involvement in MDA advocacy campaigns with knowledge on urinary schistosomiasis $(\mathrm{N}=488)$

\begin{tabular}{lllll}
\hline $\begin{array}{l}\text { Involvement in } \\
\text { advocacy campaigns }\end{array}$ & MDA & \multicolumn{2}{l}{ Level of knowledge } & \\
& Low & Medium & High & Total \\
\hline Yes & $12(30.8 \%)$ & $5(71.4 \%)$ & $243(55.0 \%)$ & $260(53.3 \%)$ \\
No & $27(69.2 \%)$ & $2(28.6 \%)$ & $199(45.0 \%)$ & $228(46.7 \%)$ \\
Total & $39(8.0 \%)$ & $7(1.4 \%)$ & $442(90.6 \%)$ & $488(100 \%)$ \\
\hline
\end{tabular}

$X^{2}$ test $=9.740, \mathrm{df}=3: p=0.021$

\section{Cercarial shedding from fresh water Bulinus snails}

A total of 46 fresh water snails were collected from 3 different ponds. Sample snails from Bahi Centre pond shed 4 cercariae; those from Nagulo Bahi pond shed 13 cercariae while those from Uhelela pond shed 8 cercariae indicating continuity of S. haematobium transmission. The incubation time was 2, 4 and 3 in Bahi Centre, Nagulo, and Uhelela, respectively. 


\section{Discussion}

The prevalence of Praziquantel MDA uptake by primary schoolchildren of 39.5\% (2011) and 43.6\% (2012) shows that the coverage is far below the WHO goal for schistosomiasis control that emphasize the coverage of between $75 \%$ and $100 \%$ of all schoolchildren at risk of morbidity by 2010 (Taylor, 2008). The low coverage observed in this study might be attributed by the perceived misconceptions on the safety of Praziquantel delivery under the School Health Programme as demonstrated in the early years of MDA implementation (D. Tarimo \& P. Mmbuji 2005 unpubl.).This might have prompted some parents not to allow their children to participate in subsequent MDA rounds including the 2011 and 2012 rounds. For the control of schistosomiasis, it is unequivocal that the coverage observed in this study fell short of Resolution 54.19 of the World Health Assembly (WHA 54.19) minimum target of treating at least $75 \%$ of all school-aged children from the endemic areas by year 2010. There is therefore the need for strengthening sensitization and awareness creation to effectively trickle through community leaders, and eventually parents and the general community so as to increase uptake of Praziquantel MDA in subsequent rounds.

Praziquantel MDA had a positive impact on reducing the prevalence of $\mathrm{S}$. haematobium, thus prevalence significantly $(p=0.000$ ) dropped by $50.0 \%$ (from an average of $30.0 \%$ microscopic prevalence before MDA to $15.0 \%$ ) after the two rounds. These results are in agreement with the findings in Burkina Faso in which a single round of Praziquantel treatment significantly reduced prevalence of the infection (Touré et al.,2008). Likewise in Mali a significant reduction of infection from baseline was observed after repeated Praziquantel treatment among school children (Landoure' et al., 2012). Uptake of Praziquantel MDA also had positive impact on the intensity of S. haematobium infection in that moderate and heavy intensity of infections were observed in only those children who did not participate in the two MDA rounds. Since treatment does not prevent or protect against subsequent infections, if water contact is continued, re-infection can take place relatively quickly (Stothard et al., 2013). The significant reduction in both prevalence and intensity might be attributed to the effectiveness of Praziquantel against $S$. haematobium where treated school children were completely cured and prevented from shedding viable eggs in the environment. Despite significant reduction, the observed S. haematobium infection prevalence of $15.0 \%$ is still within the community category for annual Praziquantel MDA to all school age-children (WHO, 2002) hence the need for further health promotion campaigns so as to increase uptake of Praziquantel MDA in subsequent rounds.

The fact that all children reported to get water for domestic use from more than one source of water indicates that there is a potential for contact with some of the water sources that may not be safe such as ponds, rivers and dams; and irrigated rice fields that may be infested with cercarial shedding Bulinus spp. snails hence the reported continuity of S. haematobium transmission. The MDA advocacy campaigns did not significantly change behaviours towards contact with water sources that may not be safe indicating that change of behaviour is a long and involved process, particularly when such behaviour has a socio-cultural or occupational nature and there are logical reasons for it (WHO, 2008). Although safe sources of water for domestic use such as wells and taps were available in the study area even before the introduction of Praziquantel MDA campaigns indicates that many factors are behind the persistent contact with potentially unsafe water sources, even when a safe water source is available (Watts et al.,1998). Thus contact with ponds, rivers and dams may be continued for play purposes while contact with irrigated rice fields is continued for occupational reasons. Kosinski et al., (2012) demonstrated that installation and use of water recreation areas as alternative source of safe water for recreation was feasible and highly effective means to reduce the incidence of schistosomiasis in school-aged children.

Knowledge about urinary schistosomiasis is an important component of Praziquantel MDA 
campaigns that need to be evaluated so as to assess its impact on the change of behaviours related to schistosomiasis transmission and control. Effective MDA advocacy campaigns are likely to improve knowledge about urinary schistosomiasis as shown in this study whereby the majority of the school children had high level of knowledge about the disease. Our findings are similar to what was reported in Kinondoni in Tanzania, however, in the later study knowledge about treatment was very low conceivably because the study was carried before the Praziquantel MDA campaigns (Ndyomugyenyi \& Minjas, 2001).

We also detected positive impact of MDA campaigns on children's level of knowledge on mode of S. haematobium transmission and therefore re-infection. Health promotion messages about prevention (for avoidable contact with potentially unsafe water sources) and treatment with Praziquantel (for unavoidable contacts with potentially unsafe water sources for occupation or recreational reasons) might explain these observations (Moji et al., 1998). This shows that the Praziquantel MDA advocacy campaigns imparted knowledge on S. haematobium transmission and reinfection that changed their behaviour consequently leading to a reduction in infection prevalence. Effective health promotion on schistosomiasis transmission has been shown to induce effective behavioural change consequently leading to a reduction in re-infection and therefore prevalence of urinary schistosomiasis (Moji et al., 1998).

A total of 46 Bulinus spp. snails, the principal intermediate hosts were sampled from three different water bodies associated with human water contacts indicating the potential for $S$. haematobium transmission. The fact that the 6 snails subjected to cercarial shedding experiment were found shedding schistosome cercariae indicates that these snails were sampled from water bodies contaminated with schistosome eggs. This shows that contamination of water bodies with schistosome eggs is still ongoing. Thus despite the high knowledge on urinary schistosomiasis displayed by the school children, they were still contaminating the surrounding water bodies with schistosome eggs hence the continuity of infection and re-infection in the study area. Schistosomiasis is essentially tied to local water-use behaviours (King, 2001). Previous studies have indicated that school children engage in more high-risk behaviours when in contact with water (Klumpp \& Webbe, 1987), in so doing either acquire the infection or contaminate the water bodies with eggs thus causing the continuity of infection and re-infection. The continuity of infection transmission and re-infection among schoolchildren in the study area may conceivably be explained by the low uptake of MDA as clearly shown that for both the two years 2011 and 2012 uptake was by far below $50.0 \%$. Thus infected schoolchildren who did not participate in the MDA rounds would continue excreting $\mathrm{S}$. haematobium eggs in the environment consequently creating new infections or re-infections. The findings therefore call for consideration of snails control to be integrated with MDA campaigns so as to achieve transmission interruption.

Though MDA significantly reduced prevalence of S. haematobium, uptake was below $50.0 \%$ and below the World Health Assembly resolution 54.19 target of $75.0 \%$ for 2010 . Non-participation in MDA was the likely source of $S$. haematobium eggs in the environment hence the observed schoolchildren with S. haematobium infection and cercarial shedding Bulinus spp. snails indicating continuity of transmission. Further health promotion campaigns are necessary so as to increase uptake of MDA rounds and to achieve transmission interruption.

\section{Acknowledgements}

We are thankful to the Bahi district authorities for their co-operation and granting us permission to carry out this work. Thanks are due to the teachers of the respectful schools for their permission and assistance to carry out this work in their schools. Special thanks to parents for their informed consent for their children to participate in the study. Special thanks to the schoolchildren for their 
acceptance and participation in the study. This study received financial support from the Directorate of Postgraduate Studies, Muhimbili University of Health and Allied Sciences.

\section{References}

Fransden, F. \& Christensen, N.Ø. (1984) An introductory guide to the identification of cercariae from African freshwater snails with special reference to cercariae of trematode species of medical and veterinary importance. Acta Tropica 41, 181-202.

King, C.H. (2001) Epidemiology of schistosomiasis: determinants of transmission of infection. A.A.F, Mahmoud ed. Schistosomiasis. London: Imperial College Press, 115-132.

Klumpp, R.K. \& Webbe, G. (1987) Focal, seasonal and behavioural patterns of infection and transmission of Schistosoma haematobium in a farming village at the Volta Lake, Ghana. Journal of Tropical Medicine \& Hygiene 90, 265-281.

Kosinski, K.C., Adjei, M.N., Bosompem, K.M., Crocker, J.J., Durant, J.L., Osabutey, D., Plummer, J.D., Stadecker, M.J., Wagner, A.D., Woodin, M. \& Gute, D.M. (2012) Effective Control of Schistosoma haematobium Infection in a Ghanaian Community following Installation of a Water Recreation Area. PLoS Neglected Tropical Diseases 6(7): e1709.

Kristensen, T.K. (1987) Danish Bilharzia Laboratory, A field Guide to African freshwater snails, East African species, 2nd edition.

Landouré, A., Dembélé, R., Goita, S., Kané, M., Tuinsma, M., Sacko, M., Toubali, E., French, M.D., Keita, A.D., Fenwick, A., Traoré, M.S. \& Zhang, Y. (2012) Significantly Reduced Intensity of Infection but Persistent Prevalence of Schistosomiasis in a Highly Endemic Region in Mali after Repeated Treatment. PLoS Neglected Tropical Diseases 6: e1774.

MoHSW (2012) Tanzania Mainland Strategic Master Plan for the Neglected Tropical Diseases Control Program 2012-2017. Ministry of Health \& Social Welfare, Dar es Salaam, Tanzania.

Moji, K. (1998) Health Education Approaches to Control Urinary Schistosomiasis in Developing Countries. Acta Medica Nagasakiensia 43, 1-11.

Ndyomugyenyi, R. \& Minjas, J.N. (2001) Urinary schistosomiasis in schoolchildren in Dar-es-Salaam, Tanzania, and the factors influencing its transmission. Annals of Tropical Medicine \& Parasitology 95, 697-706.

SCl/MoHSW (2010) Schistosomiasis Baseline Survey Report. SCl/MoHSW/WHO, Dar es Salaam, Tanzania.

Stothard, J.R., Sousa-Figueiredo, J.C. \& Navaratnam, A.M.D. (2013) Advocacy, policies and practicalities of preventive chemotherapy campaigns for African children with schistosomiasis. Expert Review of Anti- Infective Therapy 11: 733-752

Taylor, M. (2008) Global trends in schistosomiasis control. Bulletin of the World Health Organization 86, 738.

Touré, S., Zhang, Y., Bosqué-Oliva, E., Ky, C., Ouedraogo, A., Koukounari, A., Gabrielli, A.F., Sellin, B., Webster, J.P. \& Fenwick, A (2008) Two-year impact of single praziquantel treatment on infection in the national control programme on schistosomiasis in Burkina Faso. Bulletin of the World Health Organization 86, 780-787.

Watts, S., Khallaayoune, K., Bensefia, R., Laamrani, H. \& Gryseels, B. (1998) The study of human behavior and schistosomiasis transmission in an irrigated area in Morocco. Social Science \& Medicine 46, 755-765.

WHO (1991) Basic Laboratory Methods in Medical Parasitology. World Health Organization, Geneva.

WHO (2002) Prevention and Control of Schistosomiasis and Soil-Transmitted Helminthiasis. Report of a WHO Expert Committee, WHO Technical Report Series 912.

WHO (2008) The Social Context of Schistosomiasis and its Control: An Introduction and Annotated 
Bibliography. World Health Organization, Special Programme for Research and Training in Tropical Diseases, Geneva; Chapter 3: pp 49-59.

WHO (2012) Schistosomiasis: population requiring preventive chemotherapy and number of people treated in 2010 and Estimates of the population requiring preventive chemotherapy for schistosomiasis annually. Weekly Epidemiological Record 87, 37-44. 